\title{
Atitudes em relação à matemática em estudantes de Administração
}

\author{
Verônica Lídia Peñaloza Fuentes \\ Ronaldo Lima \\ Diego de Sousa Guerra
}

\begin{abstract}
Resumo
Esta pesquisa objetivava conhecer a predisposição dos alunos de Administração em relação à Matemática, bem como o enfoque utilizado em seus estudos. Espera-se contribuir com instrumentos de diagnóstico que permitam pesquisar o ambiente de aprendizagem da sala de aula. As técnicas utilizadas foram análise fatorial, estatística descritiva, testes não paramétricos e regressão binária logistic. As escalas de atitude relacionadas à Matemática e ao enfoque de estudo foram validadas, mostrando-se consistentes. Os resultados dos testes sugeriram que diferenças de gênero, idade ou mesmo estar cursando ou não a disciplina de Matemática não explicariam qualquer atitude negativa. Contudo, os testes mostraram-se significativos com relação à variável área de conhecimento preferida antes da faculdade. Os resultados mostraram também uma associação direta entre atitude positiva e enfoque de aprendizado profundo, porém essa relação mostrou-se estatisticamente não significativa. Realizou-se uma pesquisa descritiva, direta, usando dados de corte transversal, método de survey e coleta estruturada de dados.
\end{abstract}

Palavras-chave: Ensino da matemática, ambiente da sala de aula, atitudes do estudante.

\section{Attitudes related to Mathematics of Business student}

\begin{abstract}
This study investigates the disposition of Business students related to Mathematics, and the focus used in their studies. We expect to contribute to studies about the learning environment of the classroom. The techniques used were factorial analysis, descriptive statistics, and non-parametric tests in binary logistic regression. The attitude scales related to mathematics and the focus of study have been validated by showing them up consistently. The test results suggested that differences in gender, age or even the fact of taking or not the mathematics course cannot explain any negative attitude. However, the tests were significant in relation to the preferred variable area of knowledge before the university. The results also showed a direct association between positive attitude and focus of profound learning, although this relationship was shown to be statistically non-significant. This is a descriptive direct research, and we used data cross-sectional method of survey and collection of structured data.
\end{abstract}

Keywords: Mathematics education, classroom environment, students attitudes.

\section{Actitudes en relación a la matemática en estudiantes de Administración}

\begin{abstract}
Resumen
Esta investigación tenía por objetivo conocer la predisposición de los alumnos de Administración en relación a la matemática, así como también el enfoque usado en sus estudios. Se espera contribuir con instrumentos de diagnóstico que permitan investigar el ambiente de aprendizaje del salón de clase. Las técnicas utilizadas fueron de análisis factorial, estadística descriptiva, pruebas no paramétricas y regresión binaria logística. Las escalas de actitud relacionadas a la Matemática y al enfoque de estudios fueron validadas, mostrándose consistentes. Los resultados de las pruebas sugirieron que las diferencias de género, edad o inclusive estar cursando o no el curso de Matemática no explicarían cualquier actitud negativa. Sin embargo, las pruebas se mostraron significativas con relación a la variable área de conocimiento preferida antes de la universidad. Los resultados mostraron también una asociación directa entre actitud positiva y enfoque de aprendizaje profundo, pero esa relación se mostró estadísticamente no significativa. Se realizó una investigación descriptiva, directa, usando datos de corte transversal, método de Survey y recolección estructurada de datos.
\end{abstract}

Palabras-clave: Enseñanza de matemática, ambiente de la classe, actitudes del estudiante. 


\section{Introdução}

A Teoria da Administração tem recebido muitas influências das ciências exatas, especificamente da Matemática. Modelos matemáticos têm proporcionado soluções a diversos problemas e/ou situações empresariais na área de pessoal ou Recursos Humanos, na área de produção, de Marketing e principalmente na área de Finanças.

O administrador tem a seu alcance inúmeras ferramentas que facilitam o processo de tomada de decisão nas organizações. Algumas em destaque são: a programação linear, modelos de prognóstico e modelos financeiros, mas não só, há uma série de instrumentos quantitativos que se tornam cada vez mais importantes na formação de um administrador e que requerem uma boa base matemática. Outrossim, além de fazer parte de todo o patrimônio cognitivo da Humanidade (Santos, Capelari, \& Sperandio, 1998), o ensino de Matemática é enriquecedor do ponto de vista do conhecimento intelectual, pois faz uso do pensamento lógico, do demonstrativo, do intuitivo, do criativo, da imaginação e do raciocínio, características essenciais ao administrador.

Atualmente é requerido de um administrador o desenvolvimento do raciocínio lógico numérico. Este será aplicado no cotidiano em questões como: saber estruturar variáveis, criar e gerenciar projeções de valores no tempo, conhecer as principais metodologias de avaliação de investimentos, compreender o conceito de risco/retorno e quantificá-lo etc. Em diversas decisões administrativas, principalmente nas financeiras, é possível a representação dessas situações por intermédio de equações matemáticas e, então, proceder o estudo do comportamento pretérito, presente e futuro das várias situações através de simulações hipotéticas e reais.

Com o passar do tempo, a demanda pelo domínio da Matemática aumentou. $\mathrm{O}$ uso da tecnologia tem permitido grandes mudanças no ambiente de pesquisa acadêmicoaplicada e no contexto empresarial, mais especificamente no âmbito dos negócios, em que há uma diversificação de uso de ferramentas e aplicações.

Além da explosão de dados e do desenvolvimento de instrumentais, os avanços computacionais provocaram mudanças tais que, hoje, praticamente qualquer técnica e/ou banco de dados está disponível ao alcance do computador pessoal. Neste contexto, é mister que o administrador esteja cada vez mais capacitado para a utilização de instrumental matemático e estatístico que lhe dêem suporte à tomada de decisão nos campos de conhecimento da Administração.

Não obstante, observa-se ainda, em muitos casos, a predisposição negativa de muitos alunos do curso de administração com relação às disciplinas quantitativas e especificamente à Matemática. Frequentemente, professores das disciplinas de Matemática, por exemplo Matemática Financeira, deparam-se com essa barreira inicial, manifestada através de comentários como: "nunca me dei bem com Matemática", "a Matemática é maçante", "estudei Administração porque pensei que não veria Matemática”, "a Matemática é difícil para mim" etc. Entretanto, muitas das dificuldades em disciplinas quantitativas (Matemática/ Estatística) podem não ser resultado de aptidão insuficiente, mas reflexos de fatores de atitude com que a disciplina é enfrentada. A falta de motivação, produto de atitudes negativas com que o estudante enfrenta a disciplina, pode auxiliar ou atrapalhar a aprendizagem e afetar seu desempenho. Estes sentimentos, considerados num âmbito mais ampliado como "ansiedade" com relação à disciplina, provocam receio e desconfiança, dificultando a tarefa do professor.

O método de ensino inadequado e a falta de motivação podem vir a ser grandes obstáculos para o ensino e a aprendizagem da matemática. Para Maggi (2002), as dificuldades apresentadas pelos alunos nas disciplinas de matemática nos cursos de administração correspondem, na sua maioria, à concepção de objeto de estudo vigente no ensino tradicional. Esta concepção coloca o aluno em contato com um saber tido como acabado e não leva em consideração o contexto em que a aprendizagem ocorre e nem as diversas relações entre as disciplinas de um curso. Segundo o autor, um aluno habituado a este tipo de aprendizagem, quando entra em contato com uma situação de aprendizagem em que a sua capacidade de raciocinar de maneira lógica e formal se faz necessária e em que é solicitado a realizar avaliações qualitativas e quantitativas através de um ensino baseado em situações-problemas, apresenta algumas dificuldades em efetuar a transição de um modo de ensino, o tradicional vigente, para outro, o ensino baseado em situações-problemas.

Para selecionar estratégias de aprendizagem mais adequadas, além da natureza do conteúdo a ser desenvolvido, devem ser levadas em conta as características dos alunos. Os métodos de ensino deveriam motivar os estudantes, valorizar as diferenças individuais e, principalmente, levar em consideração as emoções negativas que provocam bloqueios na aprendizagem. Existe uma grande quantidade de estudos, pesquisas e teorias relacionadas à aprendizagem humana. Nosso objetivo, aqui, não é discutir essa temática em profundidade, interessa-nos a práxis da relação ensino-aprendizagem na área de administração e também nos interessa especificamente detectar se existem essas "emoções negativas" com relação à disciplina de Matemática Financeira.

\section{Marco Referencial}

\section{Atitude em relação à matemática}

Para Gonzalez-Pienda e cols. (2006), um aspecto que pode influenciar a aprendizagem do estudante é a atitude. Quando os professores criam um ambiente de aprendizagem em que os estudantes se sentem confortáveis e confiantes, são realçadas as atitudes positivas em relação à disciplina em questão. A criação de um ambiente de aprendizagem positivo impacta as atitudes do estudante e sua aprendizagem. 
Os estudantes que se sentem confiantes e confortáveis no ambiente de aprendizagem estão mais propícios a mostrar maior interesse na tarefa de aprendizagem. As crenças de uma pessoa sobre um determinado objeto determinam como a pessoa se sente em relação ao objeto (atitudes); é a atitude mediada pelos valores que determina as intenções de comportamento em relação ao objeto. Assim, se um aluno acredita que estudar estatística é estimulante e que será útil para sua vida, ele tenderá a apresentar atitudes positivas em relação à estatística e apresentará um comportamento pró-ativo para com esta (Silva, Brito, Cazorla, \& Vendramini, 2002).

Inicialmente, o termo atitude significou "aptidão" ou "adaptação", com um sentido de conotação física e, mais tarde, ampliou-se a ideia do termo, sugerindo também uma preparação mental para a ação. É importante destacar que há uma série de subconceitos contidos no conceito principal, conceitos tais como: Qualidade Avaliativa, no sentido de gostar e não gostar; Predisposição, no sentido de prontidão; Influência Social, as atitudes são aprendidas de muitas maneiras e a influência social é integrante do processo; e Experiência, atitudes são aprendidas da experiência e, portanto, podem ser ensinadas (Gonçalez, 2002; Ruffell \& Allen,1998). Associando o termo à Matemática, uns dos primeiros autores a falar em atitude em relação à Matemática foram Aiken e Dregen (1961), os quais afirmaram que a "Mathemaphobia" ou medos pronunciados na presença da aritmética e da Matemática e outras atitudes negativas para a Matemática exigem uma explanação. Para os autores, a explanação mais simples era que tais reações resultavam das experiências específicas na aprendizagem da Matemática e que a forma como os professores e os pais instruíam crianças na Matemática era a determinante preliminar de suas atitudes para este assunto, denominada por eles como "atitudes matemáticas".

Uma questão que se observa nos trabalhos de atitude em relação à Matemática e ou Estatística é que, no lugar das definições conceituais explícitas, a grande maioria dos pesquisadores tende a focalizar a atenção em medidas operacionais de atitudes e de ansiedade da Matemática e Estatística. Neste sentido, Hannula (2002) alerta que muitas pesquisas foram realizadas tratando da atitude em relação à Matemática, mas teoricamente o conceito não foi desenvolvido. Para ele, atitude em relação à Matemática é uma construção ambígua, usada frequentemente sem definição apropriada e que apresenta problemas pela discrepância entre a disposição emocional e a opinião, crítica usada referindo-se aos testes escritos. Di Martino e Zan (2001) distinguem duas aproximações básicas ao definir a atitude em relação à Matemática. A primeira, uma definição simples que a descreve "como o grau de afeto associado com Matemática”, ou seja, a atitude seria uma disposição emocional para a Matemática. Um segundo tipo de definições distinguiria entre a resposta, a opinião e o comportamento emocional enquanto componentes da atitude. Esta segunda aproximação é vista como incompatível com a visão extensamente aceita da atitude, das emoções e da opinião como pertencendo ao domínio afetivo.
Focalizar a atenção em medidas operacionais de atitudes e de ansiedade da Matemática tem sua explicação, já que esses tipos de trabalhos são usados como diagnóstico para posteriormente criar condições de mudança de atitude que facilitem o aprendizado do indivíduo. Uma das variáveis mais estudadas na literatura para explicar as diferenças de atitude em relação à matemática é o gênero. Para GonzalezPienda e cols. (2006), ainda que o interesse pelo estudo das atitudes e de seu papel determinante na aprendizagem da Matemática tenha sido objeto de investigação há mais de 50 anos, atualmente, a pesquisa ainda se centra sobre as diferenças das atitudes das mulheres e dos homens, bem como sobre as causas de tais diferenças. Segundo o autor, no geral, os estudos realizados apontaram que as mulheres se percepcionam como menos competentes que os homens, embora estes estudos não sejam conclusivos. Alguns autores vão mais longe, assinalando que os homens tendem a se beneficiar mais das experiências da ciência na faculdade do que as mulheres e que a expectativa do professor é que as mulheres tenham um desempenho menor em termos da participação e na realização na ciência (Kumar \& Morris, 2005). Segundo Hannula (2002), uma visão geral do desenvolvimento das atitudes durante o processo de educação formal é documentada através de "surveys" e meta-análises, que mostram diferenças de atitudes em relação à Matemática por gênero (mulheres tendem a ter uma atitude mais negativa que os homens) e também mostram que, à medida que se progride na educação formal, a atitude negativa em relação à Matemática aumenta, sendo a idade um fator decisório. Contudo, outros autores concluíram que diferenças de gênero não são estatisticamente significantes no relacionamento entre a atitude em relação à Matemática. Os resultados de Mills (2004), num estudo realizado em relação à Estatística, mostram que é mais provável que os homens não se atemorizem com a Estatística e se sintam mais confiantes quanto ao tema, mas não se observou nenhuma diferença de gênero nos resultados de desempenho.

Embora uma variedade de técnicas de medida tenha sido usada para explorar as atitudes, são comuns na literatura escalas tipo Thurstone/Likert, que utilizam uma série de multi-itens e focalizam dimensões tais como a apreciação, a avaliação e a autoconfiança em relação à Matemática. No Brasil, a escala mais utilizada é a desenvolvida por Aiken e Dreger (1961). Esta foi traduzida e validada por Brito (1998), validada e adaptada por Cazorla, Silva, Vendramini e Brito (1999) para a Estatística e posteriormente utilizada por Silva e cols. (2002) com relação à Estatística e à Matemática.

Como falado anteriormente, a questão que norteia muitos dos estudos sobre atitude é trabalhar as reações afetivas com a Matemática para gerar uma mudança nas atitudes em relação à disciplina, transformando-as em atitudes positivas. Porém, para que isso ocorra, é necessário que o professor da disciplina esteja motivado para aplicar estratégias estimulantes (Brito, 1998). Philippou e Christou (1998) realizaram um projeto visando mudar a perspectiva de um grupo de professores com relação à Matemática. O programa consistia em palestras abordando a história 
da Matemática e seus métodos. Depois do período de execução, os resultados indicaram uma melhoria significativa das atitudes, particularmente sobre a satisfação quanto à utilidade da Matemática. Se o impacto potencial de fatores afetivos na aprendizagem da Matemática é do interesse particular dos pesquisadores, também deveria ser dos educadores e dos docentes. Silva e cols. (2002), referindose ao ensino de Estatística, assinala que os educadores deveriam se preocupar mais com os aspectos afetivos do processo ensino-aprendizagem, buscando identificar a ansiedade e a atitude do aluno, para propor estratégias que visem reduzir ou eliminar esses aspectos negativos. Uma das estratégias propostas pelo autor é verificar, no início de uma disciplina, qual é a prontidão do aluno para realizála, bem como verificar no final do curso como ele se sente após realizá-la. Concordamos com o autor que é necessário conhecer a predisposição inicial dos alunos que cursam a disciplina de Matemática, Matemática Financeira neste caso. Esse é um dos objetivos deste trabalho, possibilitar o diagnóstico da predisposição e então relacioná-lo com a forma de aprendizagem dos alunos.

\section{Enfoque de Aprendizado}

Na hora de aprender, os alunos apresentam diferenças individuais, diferenças analisadas desde perspectivas distintas ou mesmo através de constructos diferentes, sendo estilo e enfoques de aprendizado os mais conhecidos. $\mathrm{Na}$ Psicologia educativa, circunscrita ao paradigma cognitivo, o interesse em estudar o processo de aprendizagem nos alunos deu origem, dentre outras linhas de pesquisa, a duas abordagens distintas: a que estuda os estilos de aprendizado e a que tem como tema central os enfoques de aprendizado Hernandez-Pina, 1993).

Segundo Marton e Sajlo (citado por Cano, 2000), os alunos tendem a adotar um modo de aprendizado (enfoque) que depende da percepção da situação de aprendizagem, ou seja, da motivação para aprender. Tomando como referência os trabalhos pioneiros de Marton \& Saljo (1976a, 1976b), assim como os aportes de Biggs (1987a, 1988, 1993) e de Entwistle (1988), os enfoques de aprendizado determinam como os processos de aprendizagem surgem das percepções dos estudantes das tarefas acadêmicas, influenciadas por suas características pessoais (Arias e cols., 2000). Há portanto, uma tensão entre o autoconceito do indivíduo e o ambiente/contexto a sua volta. Nesta tensão, aspectos relacionados à conduta humana são identificáveis, chama-se a atenção para o caráter propositivo e intencional. Estes fatores dirigem a conduta humana dentro do âmbito acadêmico, influenciam o autoconceito e a percepção das tarefas a serem realizadas pelo estudante, mas não só, influenciam também as atitudes, o interesse, as expectativas e os vários modelos mentais criados pelo estudante e usados como plano de metas para a aquisição do conhecimento. Não obstante, os fatores sofrem também influência do ambiente/ contexto, em que o professor, o conteúdo abordado, as mensagens a serem passadas aos alunos, tudo, dentro do processo de ensino/aprendizagem, tem imensa relevância na conduta humana (Canabach, Arias, Pérez, \& GonzálezPienda, 1996).

Quando, por exemplo, se discute enfoque, segundo Valle (2000), é possível separar os indivíduos em dois grupos distintos. Um dos grupos, rotulado de enfoque profundo, é composto por indivíduos inclinados a mostrar preferência por tarefas desafiadoras, com maior complexidade e alto nível de dificuldade. Embora os riscos de se dar cabo a tarefas desta natureza sejam altos, os resultados possíveis advindos de tais empreitadas são maiores e podem ser traduzidos em maior aprendizado e consequentemente aumento de conhecimento. Não obstante, o grupo rotulado de enfoque superficial tende a preferir tarefas com grau de dificuldade médio a fácil.

O marco teórico dos enfoques de aprendizado desenvolveu-se, inicialmente, numa vertente qualitativa impulsionada por Marton e Säljö e, posteriormente, numa vertente quantitativa. É nesta que se integram os estudos desenvolvidos por Entwistle, no Reino Unido, e por Biggs, na Austrália. Estes últimos investigadores desenvolveram inventários dos processos de aprendizagem procurando avaliar o constructo abordagem profunda e superficial, assumido como a forma habitual dos alunos enfrentarem o seu estudo em geral. Biggs, especificamente, desenvolveu dois questionários adaptados ao ensino universitário e secundário, respectivamente o SPQ (Students Processes Questionnaire) e o LPQ (Learning Processes Questionnaire), (Rosário, Almeida, Nuñez, \& González-Pienda, 2004).

Os trabalhos de Biggs sugeriram uma terceira abordagem à aprendizagem, o "enfoque estratégico" ou de alto rendimento. Os estudantes que adotam esta aproximação são caracterizados por usar as estratégias de aprendizagem, acreditando obter aprovação em testes e provas e, assim, alcançar seus objetivos. A dimensionalidade desse instrumento foi discutida na literatura, perguntando-se se os três fatores (superficial, profundo e de alto rendimento) mostrariam a descrição mais apropriada do constructo. Posteriormente, numa revisão da validade e consistência do instrumento, Biggs, Kember e Leung (2001), desistiram do enfoque estratégico, ficando com o enfoque profundo e superficial. Segundo os autores, os dados obtidos reforçam a convicção de que existem duas orientações fundamentais à aprendizagem, distinguindo-se pela presença ou ausência da intenção para compreender o material a aprender, tal como foi sugerido nos estudos iniciais por Marton e Säljö. Este último instrumento é conhecido como Two-Factor Process Questionnaire (R-SPQ-2F).

A ideia da reformulação do instrumento é permitir que os professores possam contar com uma ferramenta fácil e simples para diagnóstico, permitindo uma avaliação do ambiente e do processo de aprendizagem em suas próprias salas de aula. Para Biggs e cols. (2001) a reconstrução do teste do instrumento teve como consequência uma versão final com duas escalas em destaque, resumidas em escala profunda e escala superficial. Os testes estatísticos mostraram que a versão revisada possui propriedades 
psicométricas satisfatórias, portanto, os autores encorajam o uso do instrumento, pois possibilitaria uma melhora no ensino, uma vez que permitiria que o professor pudesse avaliar o ambiente e a relação do estudante com o contexto de forma rápida e eficiente, possibilitando o ajuste do ambiente/ contexto em uma perspectiva construtiva e promovendo assim uma aproximação profunda à aprendizagem.

\section{Questões de pesquisa}

Com esse referencial estruturou-se a pesquisa, delimitando os seguintes objetivos.

- Conhecer a predisposição, atitude (positiva/negativa) em relação à Matemática dos alunos que estão cursando a disciplina de Matemática Financeira.

- Identificar se a predisposição entre os alunos que não estão cursando a disciplina e os que estão cursando é diferente.

- Conhecer qual é o enfoque de estudo adotado por estes alunos.

- Estabelecer se existe relação entre atitude em relação à Matemática e o enfoque de estudo adotado pelos alunos que cursam a disciplina.

\section{Hipóteses}

- H1: a atitude (positiva/negativa) depende de experiências anteriores, portanto a preferência dos alunos de administração pela área de conhecimento das ciências exatas antes de ingressar na faculdade deveria estar relacionada a uma atitude positiva com relação à Matemática.

- H2: alunos cursando a disciplina apresentariam uma ansiedade maior em relação à Matemática e portanto uma atitude negativa .

- H3: alunos mais velhos teriam uma atitude negativa em relação à Matemática.

- H4: atitude negativa em relação à Matemática é independente de gênero.

- H5: alunos que apresentam uma atitude negativa em relação à Matemática adotariam um enfoque de estudo superficial.

\section{Método}

Trata-se de uma pesquisa descritiva, direta, em que os respondentes conhecem o verdadeiro propósito da pesquisa com dados de corte transversal, usando método de surveye coleta estruturada de dados. O método de survey para obtenção de informações baseia-se no interrogatório dos participantes, aos quais se fazem várias perguntas estruturadas sobre seu comportamento, intenções, atitudes, percepção etc. Geralmente o questionário é estruturado visando uma certa padronização no processo de coleta de dados (Malhotra,2001).
A amostra está composta por 159 estudantes do curso de administração de empresas de uma faculdade pública da região nordeste do país. Da totalidade dos indivíduos, 84 estão cursando a disciplina de Matemática Financeira, 36 correspondem a alunos de segundo semestre (que ainda não cursam a disciplina) e 36 pertencem a turmas de sexto semestre que já cursaram a disciplina de Matemática Financeira.

\section{Instrumentos}

Para medir o enfoque de aprendizado, foi utilizado o Revised Two-Factor Process Questionnaire (R-SPQ-2F) de Biggs. Este é um instrumento elaborado e validado pelo autor que consta de 20 itens medidos através de escala de likert de 5 pontos. Os 20 itens são divididos em 2 fatores principais, denominados de "enfoque profundo" e "enfoque superficial" e estes, por sua vez, são divididos nos subcomponentes motivos e estratégias de 5 itens cada, conformando 4 subescalas: motivo profundo, estratégia profunda, motivo superficial e estratégia superficial (Biggs e cols., 2001).

Para testar a validade do instrumento, realizouse, primeiramente, uma análise fatorial (componentes principais, rotação varimax) que resultou em 2 fatores. O primeiro reunindo todos os itens correspondentes ao enfoque superficial e o segundo agrupando todos os itens correspondentes ao enfoque profundo. Ambos fatores explicam em conjunto $39,46 \%$ da variância total. Por sua vez, os coeficientes de confiabilidade (alfa de Cronbach) foram de 0,73 para a escala do enfoque profundo, de 0,72 para a escala do enfoque superficial e de 0,69 para a escala do enfoque global. Aceitamos, então, a consistência da escala, uma vez que os valores geralmente aceitos para o alfa de croanbach são de 0,7 diminuindo para 0,6 em pesquisa exploratória (Hair, Anderson, Tatham, \& Black, 2005).

Para medir a atitude em relação à Matemática, foi utilizada uma escala originalmente desenvolvida por Aiken e Dreger (1961) e traduzida e validada por Brito (1998) e posteriormente utilizada por Silva e cols. (2002) com relação à Estatística e à Matemática. Esta é uma escala de Lickert de 20 afirmações, sendo 10 positivas e 10 negativas, que tentam expressar o sentimento individual em relação à Matemática. Para as alternativas que medem as atitudes positivas, atribuíram-se os pontos de forma crescente ao grau de concordância e inversamente foram atribuídos os pontos (de forma decrescente) para as afirmações que medem as atitudes negativas. Para se obter a nota final, foram somados todos os pontos e determinada a média da amostra. De acordo com o instrumento inicial, foram consideradas atitudes positivas as de pontuação acima da média e negativas, as de pontuação abaixo da média.

A validade do instrumento foi testada via análise fatorial (componentes principais, rotação varimax). A análise deu como resultado dois fatores que explicam $68,97 \%$ da variância total. $\mathrm{O}$ primeiro incluindo todos os itens que caracterizam a atitude positiva e o segundo incluindo os itens que caracterizam a atitude negativa em relação à 
Matemática. O coeficiente Alfa de Croanbach foi de 0,96, confirmando a elevada consistência interna do instrumento.

\section{Técnica de análise de dados}

Os dados foram trabalhados com o software SPSS (Statistical Package for the Social Sciences) versão 13.0, módulos de estatística descritiva e testes não paramétricos. Utilizaram-se provas não paramétricas por serem mais adaptáveis aos estudos que envolvem variáveis com níveis de mensuração nominal e ordinal. Estas técnicas são recomendadas para verificar se as variáveis são independentes ou relacionadas e também no tratamento estatístico de dados oriundos de tabelas com dupla entrada (Martins, 2002). Também foram usados os módulos de regressão e regressão binária logística. A formulação e estimação de relações nas quais estão em jogo características qualitativas de pessoas ou objetos são melhor descritas por modelos de escolha discreta, que podem ser representados por uma variável binária que toma o valor 1 na presença da característica e valor 0 na ausência da característica estudada.

\section{Resultados e Discussão}

Segundo podemos observar na Tabela 1, há uma maior proporção de alunos com atitude positiva em relação à Matemática. Dos 150 respondentes, 87 apresentam uma atitude positiva e 63 apresentam uma atitude negativa.
Quando analisamos como se comporta a atitude referida a outras variáveis, gênero, idade, cursando ou não a disciplina e área de conhecimento preferida antes da faculdade, observamos o seguinte comportamento: com relação ao gênero, não parecem existir grandes diferenças de atitude por gênero, tanto homens como mulheres (estas numa proporção um pouco maior, 59,4\%) apresentam uma atitude positiva em relação à Matemática; com relação ao fator idade, tampouco há uma grande diferença quanto à atitude em relação à Matemática, alunos mais velhos parecem ter uma atitude positiva (proporção pouco maior) que alunos mais jovens; com relação a estar cursando ou não a disciplina de Matemática Financeira, dos que estão cursando, um percentual um pouco maior apresenta uma atitude positiva em relação à Matemática e, dos que não cursaram ainda, um percentual um pouco maior apresenta uma atitude negativa. A única variável que parece fazer uma diferença importante quanto à atitude em relação à Matemática é a preferência dos alunos quanto à área de conhecimento, aqueles alunos que preferiam a área de exatas na escola têm uma atitude positiva proporcionalmente bem maior $(79,1 \%)$ que aqueles que preferiam outras áreas.

Para saber se a relação entre as variáveis atitudes em relação à Matemática e a variável binária estar cursando ou não a disciplina existe efetivamente e que o resultado observado na Tabela 1 não tem somente caráter de aleatoriedade, aplicou-se o teste quiquadrado. Obteve-se um resultado com níveis de significância em torno de 0,23 ,

Tabela 1. Número e percentual de respondentes segundo atitude em relação à Matemática versus gênero, idade, se estão cursando a disciplina e a área de conhecimento preferida antes da faculdade

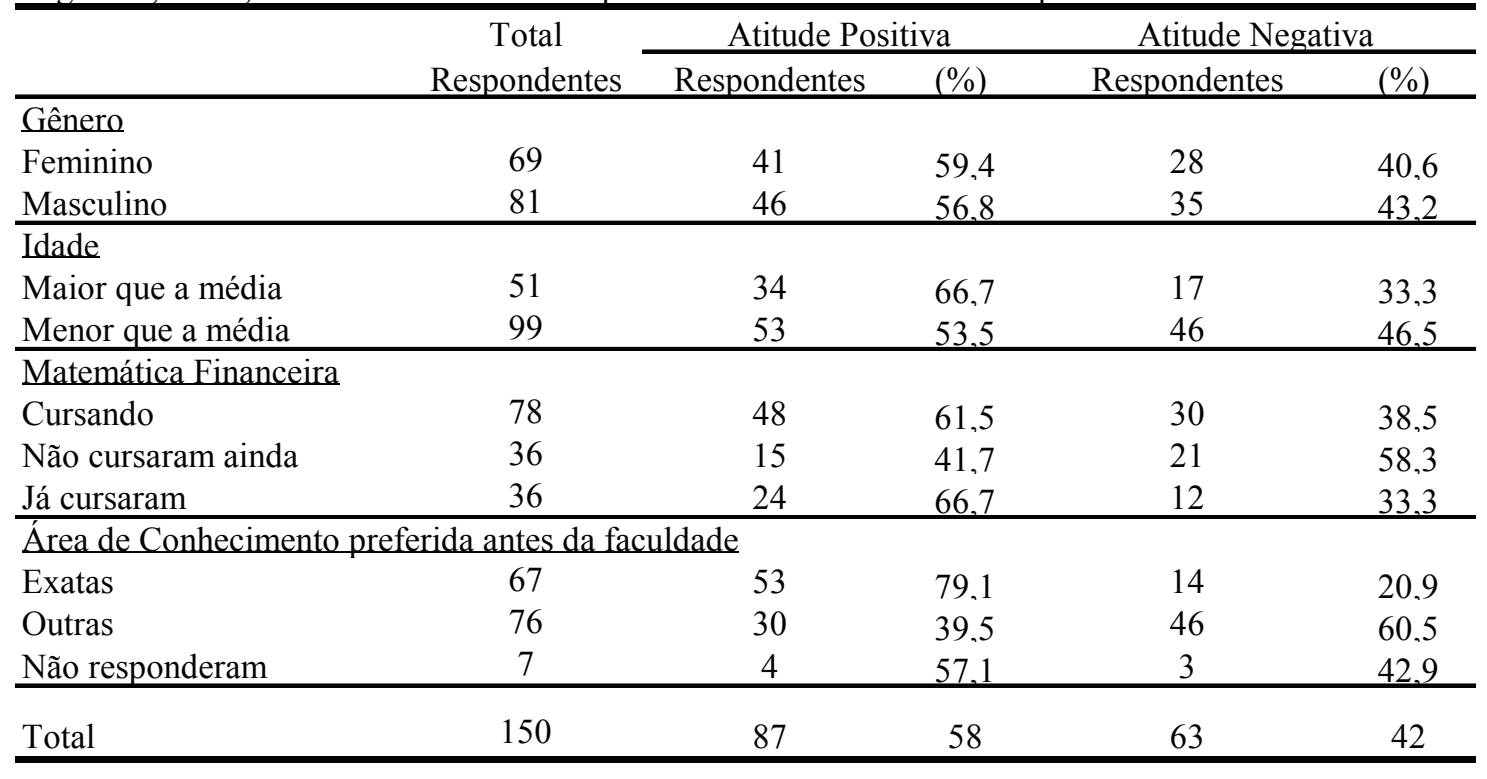

Fonte: Elaboração dos autores. 
indicando uma não existência de diferença efetiva quanto à atitude e o fato de estar cursando ou não a disciplina. No caso da variável área de conhecimento preferida na faculdade, o teste quiquadrado apresentou níveis de significância em torno de 0,00 , portanto, efetivamente existe diferença de atitude em relação à matemática e à variável área de conhecimento preferida antes da faculdade. Mais ainda, o resultado das medidas de associação, coeficiente de contingência e Phi indicaram que o grau de relacionamento entre as variáveis está em um intervalo de 37 a $40 \%$.

Confirmando os resultados acima através de regressão logistic (TAB.2), a variável atitude positiva em relação à matemática:

- Relaciona-se positivamente com o gênero feminino, embora o coeficiente não seja significativo, o que confirma a Hipótese 4, em que a atitude em relação à Matemática independe do gênero;

- Relaciona-se negativamente com as outras áreas de conhecimento que não as exatas, porém o coeficiente é significativo, confirmando desta forma a Hipótese 1;

- Relaciona-se positivamente com o fato de cursar a disciplina de Matemática Financeira, porém o coeficiente não é significativo, rejeitando a Hipótese 2, em que os alunos que cursam a disciplina teriam uma atitude mais negativa, contrariamente àqueles que não cursam a disciplina,

- Relaciona-se de forma positiva com a idade, mas o coeficiente também é não significativo, rejeitando a Hipótese 3 , em que alunos mais velhos teriam uma atitude mais negativa em relação à Matemática

Quanto ao enfoque de estudo, observamos, na Tabela 3, que 46 alunos com atitude positiva em relação à Matemática apresentam enfoque profundo de estudo e 37 alunos com atitude negativa em relação à Matemática apresentam enfoque superficial de estudos. Estes resultados sugerem a não existência de relação entre atitude e enfoque de estudos. Para corroborar esta percepção, se fez regressão entre as variáveis.

A Tabela 4 mostra que existe uma relação direta entre enfoque profundo e atitude positiva em relação à Matemática, porém o coeficiente é não significativo, rejeitando a Hipótese 5. Adicionalmente, as variáveis gênero e idade também se apresentam como não significativas em relação ao enfoque de estudo.

Tabela 2. Resutados da regressão logistic de atitude em relação à matemática

\begin{tabular}{lrrrrrr}
\hline & \multicolumn{1}{c}{ B } & \multicolumn{1}{c}{ S.E. } & \multicolumn{1}{c}{ Wald } & \multicolumn{1}{c}{ df } & \multicolumn{1}{c}{ Sig. } & Exp(B) \\
\hline genero(1) & 0,762 & 0,413 & 3,405 & 1,000 & 0,065 & 2,143 \\
AREA_PREF(1) & $-1,939$ & 0,417 & 21,653 & 1,000 & 0,000 & 0,144 \\
MAT_F(1) & 0,223 & 0,381 & 0,342 & 1,000 & 0,559 & 1,250 \\
IDADE_BI(1) & 0,589 & 0,410 & 2,066 & 1,000 & 0,151 & 1,802 \\
Constant & 0,781 & 0,421 & 3,433 & 1,000 & 0,064 & 2,183 \\
\hline
\end{tabular}

Variable(s) entered on step 1: genero, AREA_PREF, MAT_F, IDADE_BI.

Fonte: Elaboração dos autores.

Tabela 3. Número de repondentes segundo atitudes em relação a enfoques de estudo

\begin{tabular}{lccc}
\hline & Enfoque & Enfoque & \\
& Superficial & Profundo & Total \\
\hline Atitude Negativa em relação à Matemática & 37 & 25 & 62 \\
Atitude Positiva em relação à Matemática & 40 & 46 & 82 \\
Total & 77 & 71 & 148 \\
\hline
\end{tabular}

Fonte: Elaboração dos autores.

Tabela 4. Resutados da regressão logistic de enfoques de estudo

\begin{tabular}{lrrrrrr}
\hline & B & S.E. & Wald & df & Sig. & Exp(B) \\
\hline atitu_bi & 0,501 & 0,342 & 2,155 & 1,000 & 0,142 & 1,651 \\
genero(1) & $-0,260$ & 0,338 & 0,593 & 1,000 & 0,441 & 0,771 \\
IDADE_BI(1) & 0,332 & 0,355 & 0,874 & 1,000 & 0,350 & 1,394 \\
Constant & $-0,370$ & 0,322 & 1,326 & 1,000 & 0,250 & 0,691 \\
\hline
\end{tabular}

Variable(s) entered on step 1: atitu bi, genero, IDADE BI.

Fonte: Elaboração dos autores. 


\section{Considerações Finais}

A motivação deste artigo surgiu da experiência de um dos autores em sala de aula como professor da disciplina de métodos quantitativos e Matemática Financeira no curso de Administração de Empresas. A predisposição negativa com que muitos alunos enfrentam estas disciplinas desde o primeiro dia de aula, antepondo uma barreira que dificulta seu aprendizado, suscitou a pergunta sobre qual seria o melhor método de ensino a ser utilizado com estes estudantes.

Anteriormente à escolha do método de ensino a ser utilizado, era mister a identificação desses indivíduos para inferir sobre qual era a predisposição deles com relação à disciplina e tentar identificar as causas dessa atitude. Procurando um instrumental de diagnóstico, utilizou-se uma Escala de Atitudes em relação à Matemática desenvolvida por Aiken e Dreger (1961) e adaptada por outros autores para o caso brasileiro (Brito, 1998 e Silva, 2002). Validouse o instrumento, mostrando alta consistência com relação a dimensionar o sentimento que cada indivíduo possui em relação à Matemática e apurou-se que $38,5 \%$ dos alunos que estão cursando a disciplina de Matemática Financeira apresentam uma predisposição negativa em relação à Matemática. Os resultados dos testes estatísticos aplicados sugeriram que as diferenças de gênero, idade ou estar cursando ou não a disciplina de Matemática Financeira não explicariam a atitude negativa em relação à Matemática. Contudo, os testes se mostraram altamente significativos com relação à variável área de conhecimento preferida antes da faculdade. Assim, os alunos cuja área de conhecimento preferida na faculdade era ciências exatas apresentaram uma atitude positiva, em relação à Matemática, diferentemente dos alunos que preferiam as outras áreas, indicando, com isso, que a experiência anterior, dimensionada aqui pelas preferências prévias declaradas, explica a atitude em relação à Matemática manifestada pelos alunos. No caso específico desta pesquisa, a medida de associação entre essas duas variáveis seria em torno de 30 a $40 \%$.

Conjuntamente com a escala de atitudes, foi aplicado outro instrumento, o Revised Two-Factor Process Questionnaire (R-SPQ-2F) de Biggs, com intuito de apurar o enfoque de aprendizagem, profundo ou superficial, utilizado pelos alunos e de relacionar este com as atitudes em relação à Matemática. Estatisticamente, essa relação se mostrou não significativa.

Ressalta-se que a contribuição desta pesquisa limitouse à utilização e validação de instrumentos de diagnóstico, instrumentos estes que se mostraram consistentes para identificar enfoques de aprendizado e atitudes em relação à Matemática, que, todavia, até agora não tinham sido aplicados em disciplinas do curso de Administração de Empresas.

Como contribuição para futuras pesquisas, sugere-se expandir o instrumental de diagnóstico aqui proposto para outras disciplinas, por exemplo, disciplinas de estatística ou até mesmo expandir para áreas afins (como contabilidade) que ofereçam disciplinas de métodos quantitativos na sua grade curricular.
Ressalta-se, ainda, que, neste caso, só avançamos na apresentação de instrumentos de diagnóstico. Futuras pesquisas poderiam utilizar-se destes instrumentos de diagnóstico para posteriormente identificar métodos de ensino que permitam modificar as atitudes negativas dos alunos e induzir reformas da prática da sala de aula, usando formatos alternativos de ensino, permitindo que se tenha em consideração os aspectos emocionais do aluno.

\section{Referências}

Arias, A. V., Canabach, R. G., Perez, J. C. N., Riveiro, J. M. S., Aguín, I. P., \& Martínez, S. R. (2000). Enfoques de Aprendizaje en Estudiantes universitarios. Psicothema, 12(3), 368-375.

Aiken, L. R., \& Dreger, R. M. (1961). The effects of attitudes on performance in Mathematics. Journal of Educational Psychology, 52(1), 19-24.

Biggs, J. (1987). The Study Process Questionnaire (SPQ) Manual. Hawthorn: Australian Council for Educational Research.

Biggs, J. (1988) The role of the metacognition in enhancing learning. Australian Journal of Education, 32, 127-138.

Biggs, J. (1993). What do inventories os students'learning processes really measure? A theoretical review and clarification. British Journal of Educational Psychology, 63, 3-19.

Biggs, J., Kember, D., \& Leung, Y. (2001). The revised two-factor Study Process Questionnaire: RSPQ-2F. British Journal of Educational Psychology, 71, 133-149.

Brito, M. R. F. (1998). Adaptação e validação de uma escala de atitudes em relação à matemática. Zetetiké, 6(9), 109-162.

Canabach, R., Arias, A. V., Pérez, J. C. N., \& González-Pienda, J. A. (1996). Una aproximación teórica al concepto de metas académicas y su realización con la motivación escolar. Psicothema, 8(1), 45-61.

Cano, F. (2000) Diferencias de género en estrategias y estilos de aprendizaje. Psicothema, 12(3), 360-367.

Cazorla, I. M., Silva, C. B., Vendramini, C. M. M., \& Brito, M. F. R. (1999). Adaptação e validação de uma escala de atitudes em relação à estatística. Em Anais da conferência internacional: experiências e perspectivas do ensino da estatística (pp. 45-57). Florianópolis: ABE.

Di Martino, P., \& Zan, R. (2001). Attitude toward mathematics: some theoretical issues. Em M. Van den Heuvel-Panhuizen (Ed.), Proceedings of the 25th Conference of the International Group for the Psychology of Mathematics Education, Vol. 3, (pp. 209216). Utrecht, Neatherlands: Freudenthal Institute, University of Utrecht.

Gonçalez, N. (2002) Atitudes dos alunos do curso de pedagogia com relação à disciplina de estatística no laboratório de informática. Tese de Doutorado, Universidade de Campinas, Campinas, São Paulo.

Gonzalez-Pienda, J. A., Nuñez, J. C., Solano, P., Silva, E. H., Rosário, P., Mourão, R., \& Valle, A. (2006). Looking at Mathematics through gender: a study in Spanish compulsory education. Estudos de Psicologia (Natal), 11(2), 135-141.

Hair Jr., J. F., Anderson, R. E., Tatham, R. L., Black, W. C. (2005). Análise Multivariada de Dados. Porto Alegre: Bookman.

Hannula, M. (2002). Attitude towards mathematics: motions, expectations and values, Educational Studies in Mathematics, $49,25-46$. 
Hernández-Pina, F. (1993). Concepciones en el estudio del aprendizaje de los estudiantes universitarios. Revista de Investigación Educativa, 22, 117-150.

Kumar, D. D., \& Morris, J. D. (2005). Predicting Scientific Understanding of Prospective Elementary Teachers: Role of Gender, Education Level, Courses in Science, and Attitudes Toward Science and Mathematics. Journal of Science Education and Technology, 14(4), 387-391.

Maggi, L. (2002) Fatores críticos no ensino da Matemática nos cursos de Administração de Empresas: as dificuldades apresentadas pelos alunos ingressantes e as suas implicações na aprendizagem. Enangrad [On-line], 13. Recuperado: Nov. 2006. Disponível: http://www.angrad.org.br/.

Malhotra, N. (2001) Pesquisa de marketing: uma orientação aplicada. Porto Alegre: Bookman.

Martins, G. (2002). Estatística geral e aplicada. (2a ed.). São Paulo: Atlas.

Marton, F., \& Saljo. (1976a). On Qualitative Differences in Learning 1: Outcome and Process. Brit. J. Educ. Psych., 46, 4-11.

Marton, F., \& Saljo. (1976b). On Qualitative Differences in Learning - 2: Outcome as a function of the learner's conception of the task. Brit. J. Educ. Psych., 46, 115-27.
Mills, J. (2004). Students Attitudes toward Statistics: Implications for the Future. College Student Journal, 38(3), 349-361.

Philippou, G. N., \& Christou, C. (1998). The Effects of a Preparatory Mathematics Program in Changing Prospective Teachers' Attitudes Towards Mathematics. Journal Educational Studies in Mathematics, 35(2), 189-206.

Rosário, P., Almeida, L. S., Nuñez, J. C., \& González-Pienda, J. A. (2004). Abordagem dos alunos à aprendizagem: análise do construto. PsicoUSF, 9(2), 117-127.

Ruffell, J. M. M, \& Allen, B. (1998). Studying attitude to mathematics. Educational Studies in Mathematics, 35, 1-18.

Santos, A. K., Capelari, R., \& Sperandio, D. (1998). É relevante o estudo da matemática na formação do administrador contemporâneo?. Enangrad, 9. Recuperado: Nov. 2006. Disponível: http://www.angrad.org.br/.

Silva, C. B. da., Brito, M. R. F. de., Cazorla, I. M., \& Vendramini, C. M. M. (2002). Atitudes em relação à estatística e à matemática.

Psico-USF, 7(2), 219-228.

Recebido em: 02/07/2008

Reformulado em: 06/06/2009

Aprovado em: 03/07/2009

\section{Sobre os Autores}

Verônica Lídia Peñaloza Fuentes

Universidade Estadual do Ceará.

Ronaldo Lima

Universidade de São Paulo.

Diego de Sousa Guerra

Universidade Estadual do Ceará. 